\title{
Análise do Número de Linfonodos em Espécimes de Ressecções Colorretais por Neoplasia entre a Cirurgia Aberta e Videolaparoscópica
}

\author{
Comparison of the Number of Retrieved Lymph Nodes in Colorectal \\ Cancer Resection Specimens Between Conventional and Laparoscopic \\ Surgery
}

\author{
PAULO GUSTAVO KOTZE' ${ }^{1}$ CRISTIANO DENONI FREITAS ${ }^{2}$; ILÁRIO FROEHNER JUNIOR ${ }^{2}$; \\ JULIANA STRADIOTTO STECKERT ${ }^{3}$; ELISSA ISHIE $^{4}$; ÁLVARO STECKERT FILHO'; JULIANA FERREIRAMARTINS ${ }^{5}$; \\ ERONFÁBIO MIRANDA
}

1. Chefe do Serviço de Coloproctologia do Hospital Universitário Cajuru (SeCoHUC-PUCPR); ${ }^{2 .}$ Médicos residentes do SeCoHUC-PUCPR; ${ }^{3}$ Ex-médica residente do SeCoHUC-PUCPR, médica do serviço de Coloproctologia do Hospital Florianópolis - SC; ${ }^{4}$ Acadêmicos de medicina, ex-estagiários do SeCoHUC-PUCPR; ${ }^{5}$ Membros do Staff do $\mathrm{SeCoHUC-PUCPR}$.

KOTZE PG; FREITAS CD; FROEHNER JUNIOR I; STECKERT JS; ISHIE E; STECKERT FILHO A; MARTINS JF; MIRANDA EF. Análise do Número de Linfonodos em Espécimes de Ressecções Colorretais por Neoplasia entre a Cirurgia Aberta e Videolaparoscópica. Rev bras Coloproct, 2010;30(2): 119-127.

RESUMO: Introdução: o estadiamento patológico com a análise do número de linfonodos dissecados é fator importante na determinação da segurança oncológica das ressecções por câncer colorretal, independentemente da via de acesso. Em fase inicial de curva de aprendizado em laparoscopia colorretal, a equivalência entre a cirurgia convencional e laparoscópica pode ser comprometida. $O$ objetivo do presente estudo foi analisar o número de linfonodos dissecados em espécimes de ressecções por câncer colorretal pela via convencional e laparoscópica, e verificar a equivalência oncológica entre ambas. Método: estudo retrospectivo de uma série de casos de pacientes submetidos a ressecções por câncer colorretal por via convencional e laparoscópica. Variáveis analisadas: idade, sexo, via de acesso, tipo de procedimento, estadiamento de Dukes e número de linfonodos dissecados nas peças. Análise estatística pelo método de Mann-Whitney. Resultados: 50 pacientes foram analisados (33 operados por via convencional, 17 por via laparoscópica). Houve maior número de colectomias direitas e retossigmoidectomias altas nos dois grupos. O número médio de linfonodos dissecados foi de 10,35 no grupo laparoscópico e de 10,15 no grupo de acesso convencional $(p=0,859)$. Conclusões: não houve diferença estatística entre o número médio de linfonodos dissecados entre os espécimes ressecados por via convencional e laparoscópica, numa fase inicial de curva de aprendizado.

Descritores: Neoplasias Colorretais; Linfonodos; Cirurgia Colorretal; Laparoscopia.

Trabalho realizado no Serviço de Coloproctologia do Hospital Universitário Cajuru (SeCoHUC) - PUCPR - Curitiba - PR - Brasil. 
Análise do Número de Linfonodos em Espécimes de Ressecções Colorretais por Neoplasia entre a Cirurgia Aberta e Videolaparoscópica

Paulo Gustavo Kotze e Cols.
Vol. 30

\section{INTRODUÇÃO}

O câncer colorretal (CCR) é a terceira causa mais comum de neoplasias no mundo em ambos os sexos, além do segundo tumor mais frequente em países desenvolvidos ${ }^{1}$. Cerca de $9,4 \%$ de todas as neoplasias, o que corresponde a um milhão de casos novos por ano, são do cólon e reto. Nos Estados Unidos, estima-se que sejam diagnosticados 146.970 casos novos por ano, e que em 2009 ocorreram 49.920 óbitos relacionados à doença ${ }^{2}$. Já no Brasil, segundo o Instituto Nacional do Câncer (INCA), o CCR é o quarto tumor em incidência, com uma estimativa de 28.110 mil novos casos para $2010^{1}$.

A principal forma de tratamento é a ressecção cirúrgica do tumor. O prognóstico tem melhorado muito nas últimas duas décadas, devido ao diagnóstico mais precoce, avanços na radioterapia e quimioterapia e à introdução de melhorias técnicas, como a excisão do total do mesorreto, nos casos de tumores do reto médio e distal ${ }^{3}$.

$\mathrm{O}$ estadiamento patológico representa o fator prognóstico mais importante para os pacientes com câncer colorretal. O Sistema Tumor-Nódulo-Metástase (TNM), definido pela American Joint Committee on Cancer, é o sistema de estadiamento mais descrito nos estudos $^{4}$. Outros tipos de classificação, como o consagrado sistema de Dukes, também podem ser utilizados. Um dos fatores prognósticos mais importantes é o acometimento linfonodal dos tumores. Linfonodos acometidos determinam a necessidade de quimioterapia adjuvante ${ }^{5,6}$, o que salienta a importância de uma linfadenectomia bem executada pelo cirurgião e a análise acurada da peça pelo patologista, para o desfecho terapêutico otimizado.

Até a década de 90, a cirurgia convencional aberta era a única opção de tratamento cirúrgico para o CCR. Este tipo de via de acesso requer grandes incisões, apresenta grande agressividade cirúrgica, além de períodos longos de hospitalização. A cirurgia laparoscópica trouxe grande progresso no tratamento de muitas doenças do sistema digestório ${ }^{7}$. A primeira ressecção colorretal por acesso laparoscópico para CCR foi realizada por Jacobs em $1990^{8}$. Trata-se de via de acesso menos invasiva, que apresenta menor dor pós-operatória, redução na permanência hospitalar e retorno precoce às atividades pessoais ${ }^{9}$.

$\mathrm{O}$ uso da videolaparoscopia no tratamento curativo do CCR encontrou muita resistência e preocu- pação em relação ao princípios oncológicos de ressecção dos tumores, tais como: recorrência local e na cicatriz cirúrgica (principalmente no local dos portais ${ }^{10}$ ), metástases à distância e sobrevida a longo prazo. Em certa época, limitou-se a cirurgia minimamente invasiva para tratamento do CCR a estudos controlados randomizados.

Quatro grandes estudos multicêntricos compararam os desfechos do acesso laparoscópico em relação ao acesso convencional na recorrência e sobrevida do câncer de cólon - Barcelona ${ }^{11}$, Clinical Outcomes of Surgical Therapy Study Group $(\mathrm{COST})^{12}$, Conventional versus LaparoscopicAssisted Surgery in Colorectal Cancer (CLASICC) ${ }^{13}$ e Colon Cancer Laparoscopic or Open Resection $(\mathrm{COLOR})^{14}$. Estes trabalhos concluíram que há exeqüibilidade técnica da videocirurgia colorretal em obediência aos princípios da cirurgia oncológica, sem diferença em relação à cirurgia convencional. Estes estudos mostraram que a extensão da linfadenectomia, a morbimortalidade e as margens de ressecção foram semelhantes entre as vias de acesso laparoscópica e convencional. Da mesma forma, não houve diferenças na sobrevida global e na taxa de recorrência após seguimento médio de três a cinco anos.

Para o câncer do reto, sabe-se que a laparoscopia é um acesso tecnicamente possível e seguro, além de oferecer resultados a longo prazo comparáveis ao acesso convencional ${ }^{15}$. Em termos oncológicos, a ressecção total do mesorreto, a linfadenectomia e a apropriada margem distal podem ser alcançadas pela abordagem videolaparoscópica, após adequada curva de aprendizado ${ }^{16}$. No entanto, o estudo randomizado COLOR II $^{17}$ está ainda em andamento para comprovar tais desfechos.

A conquista desta equivalência entre as vias de acesso, com relação aos fundamentos oncológicos, requer alto grau de constância, destreza e habilidades técnicas pelo cirurgião. $\mathrm{O}$ alcance destas metas necessita de um período de treinamento inicial a fim da proficiência nestes complexos procedimentos ${ }^{18}$. A American Society of Colon and Rectal Surgeons (ASCRS) aprovou a realização de procedimentos laparoscópicos para neoplasias colorretais com intenção curativa, desde que haja treinamento prévio em simuladores e cursos específicos. Dados do estudo $\mathrm{COST}^{12}$ sugerem que há restrita recomendação de que pelo menos 20 ressecções colorretais laparoscópicas em doenças benignas tenham sido realizadas pelo ci- 
rurgião antes da realização da colectomia oncológica por vídeo.

Ao realizar-se a videolaparoscopia, o cirurgião eventualmente se depara com certa dificuldade de se definir o estadiamento intra-operatório. Isto ocorre porque não se pode palpar o mesocólon, na investigação de metástases de linfonodos, nem estimar sua distribuição, mesmo com boa visibilidade ${ }^{19}$. Além disso, a presença de linfonodos comprometidos justifica a indicação de terapia adjuvante, que pode determinar uma sobrevida maior. Assim, a pesquisa destes parâmetros é considerada como procedimento de maior relevância na análise histológica de espécimes operatórios em pacientes submetidos ao tratamento do $\mathrm{CCR}^{20}$.

O número mínimo de linfonodos dissecados nos espécimes ressecados de CCR, aceito pela literatura como adequado padrão oncológico, é de $12^{5,6,21}$. Tratase de um número que determina uma ressecção aceita como ampla e com linfadenectomia segura. Entretanto, há diversidade sobre este conceito entre grandes centros de pesquisa no tema.

Diante deste cenário, de antigas incertezas sobre a ressecção do CCR por meio da videolaparoscopia e da importância da pesquisa de linfonodos comprometidos como fator prognóstico, justifica-se a necessidade de mais estudos sobre o tema. Esta necessidade ocorre principalmente no nosso meio, um hospital universitário com programa de residência médica em coloproctologia, para se avaliar a performance das ressecções laparoscópicas para CCR realizadas no serviço, em fase inicial de curva de aprendizado.

O objetivo principal deste estudo foi confrontar a via laparoscópica com a via convencional, em relação ao número de linfonodos dissecados encontrados nos espécimes ressecados por CCR em um serviço universitário de coloproctologia, em sua curva inicial de aprendizado. Desta forma, verificar se há equivalência em relação a esta variável entre as duas vias de acesso. Os objetivos secundários foram avaliar dados demográficos dos pacientes submetidos ao tratamento cirúrgico para CCR pelas duas técnicas, além de avaliar os tipos de ressecções quanto à topografia da lesão, e o estadiamento dos tumores ressecados pelas duas vias.

\section{MÉTODO}

Este estudo foi aprovado previamente pelo Comitê de Ética e Pesquisas em seres humanos da
PUCPR (CEP - PUCPR), sob o número 5087/2009. Trata-se de um estudo retrospectivo, analítico e transversal, de uma série de casos. A população estudada foi constituída por pacientes do Serviço de Coloproctologia do Hospital Universitário Cajuru (SeCoHUC - PUCPR), que foram submetidos ao tratamento cirúrgico do CCR por meio de cirurgia convencional e videolaparoscópica. A coleta de dados foi feita através do acesso aos registros médicos (prontuários) dos pacientes, registrando-se vários dados e preenchimento de protocolo específico, baseado na análise do laudo anátomo-patológico dos espécimes ressecados.

As variáveis analisadas foram: idade, sexo, localização do tumor, tipo de procedimento (em relação à topografia), via de acesso, estadiamento de Dukes e número de linfonodos dissecados nas peças. O estadiamento de Dukes foi escolhido por ser definido como mais fidedigno na análise dos laudos anátomopatológicos dos espécimes.

Foram incluídos no estudo todos os pacientes operados no referido serviço entre janeiro de 2007 e maio de 2009, para ressecção de CCR, tanto pela via convencional como pela via laparoscópica. Foram excluídos os pacientes que tiveram irressecabilidade, e os que sofreram operações paliativas (operações de bypass, ou estomas de desvio, por exemplo).

Todos os dados foram coletados por um pesquisador, sendo compilados em tabelas para posteriores análises e avaliações. Todos os pacientes foram operados pela mesma equipe cirúrgica, independentemente da via de acesso realizada para a ressecção (convencional ou laparoscópica).

Os pacientes foram divididos em dois grupos: grupo de cirurgia convencional (grupo controle) e de cirurgia laparoscópica (grupo de estudo). Os grupos foram comparados em relação às variáveis estudadas, e a hipótese principal testada é a de equivalência no número de linfonodos nos espécimes ressecados pelas duas vias de acesso. Através desta hipótese, pretendeu-se saber se as operações foram realizadas com os mesmos princípios oncológicos, por via convencional ou laparoscópica.

Os valores encontrados foram armazenados em um banco de dados no programa EPIDATA. A análise estatística foi feita pelo programa SPSS versão 17.0, baseada no método de Mann-Whitney $\operatorname{com} p<0,05$ com intervalo de confiança de $95 \%$. 
Análise do Número de Linfonodos em Espécimes de Ressecções Colorretais por Neoplasia entre a Cirurgia Aberta e Videolaparoscópica Paulo Gustavo Kotze e Cols.
Vol. 30

\section{RESULTADOS}

Foram estudados no período 50 pacientes submetidos a ressecções por CCR, sendo 33 (66\%) pela via convencional e 17 (34\%) pela via laparoscópica. A média de idade para a via aberta foi 62,84 (47-90) anos e para a via laparoscópica foi de 52,23 (33-86) anos. A distribuição destes pacientes por idade em relação à via de acesso está demonstrada na figura 1.

Em relação ao sexo, do total de 50 pacientes, 25 eram homens e 25 mulheres. A figura 2 ilustra a distribuição quanto ao sexo e as vias de acesso realizadas, com predominância de homens na via de acesso convencional e de mulheres na via laparoscópica.

Em relação ao estadiamento das peças cirúrgicas ressecadas entre as duas vias de acesso, os achados encontram-se descritos na figura 3 . Houve 8 pacientes com estadiamento de Dukes A, 20 com Dukes B e 22 com Dukes C.

Ao se classificar os procedimentos cirúrgicos realizados, observou-se 15 colectomias direitas, 22 retosigmoidectomias com anastomoses altas, 4 retossigmoidectomias com anastomoses baixas e 9 amputações abdômino-perineais do reto. Estes achados em relação às vias de acesso realizadas estão ilustrados na figura 4. Não houve diferença entre os grupos em relação a colectomias direitas e retossigmoidectomias altas. O número de amputações do reto e de retossigmoidectomias com anastomoses baixas analisado foi muito pequeno, o que não permitiu uma análise adequada.

O número médio de linfonodos encontrados foi de 10,35 (mínimo de 1 e máximo de 23) na via laparoscópica e 10,15 (mínimo de 1 e máximo de 22) na via aberta, sem significância estatística $(p=0,859)$ (figura 5). Não houve, portanto, diferença estatística entre o número de linfonodos dissecados nos espécimes de ressecções colorretais entre as duas vias, o que comprovou a hipótese do estudo. Pode-se afirmar que no início da curva de aprendizado do serviço, as operações foram igualmente realizadas do ponto de vista oncológico, apenas com a diferença da via de acesso.

\section{DISCUSSÃO}

No início da década de 90 a primeira ressecção de CCR por videolaparoscopia foi realizada ${ }^{8}$. Houve rápida evolução desta via de acesso, e os conhecimen-

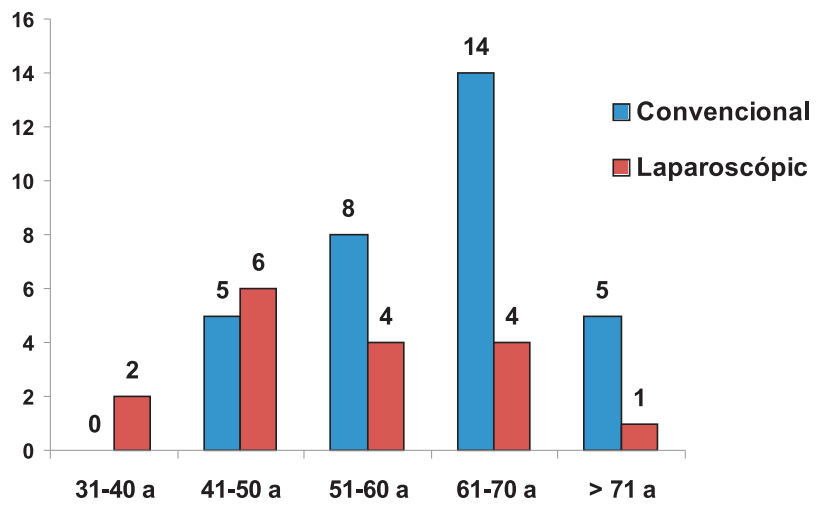

Figura 1 - Distribuição das ressecções colorretais por neoplasia quanto à idade.

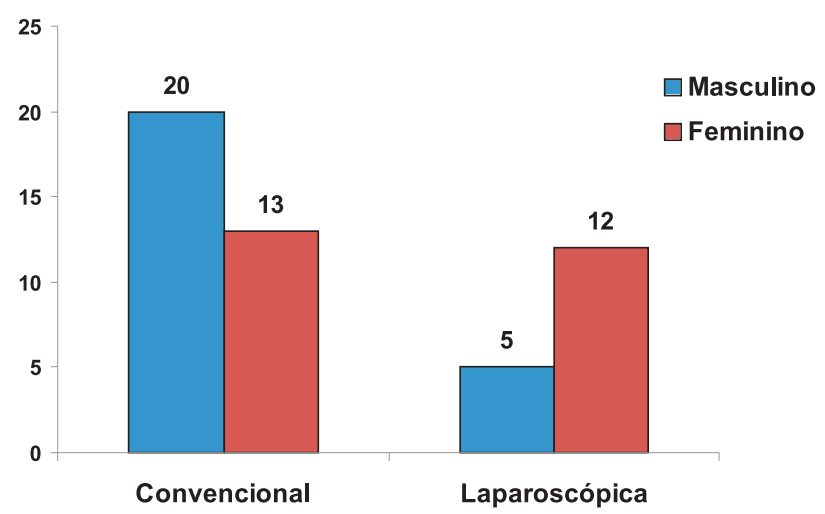

Figura 2 - Distribuição da via de acesso das ressecções em relação ao sexo.

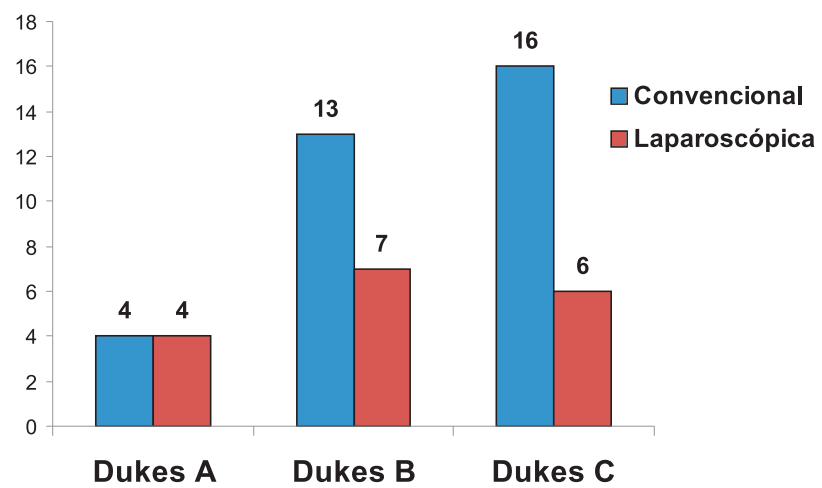

Figura 3 - Estadiamento de Dukes em relação à via de acesso cirúrgico.

tos sobre as ressecções colorretais laparoscópicas foram espalhados em nível exponencial. Desde então, alguns trabalhos randomizados com nível de evidência A foram publicados. Estes estudos comprovaram a equivalência oncológica desta via de acesso com a ci- 


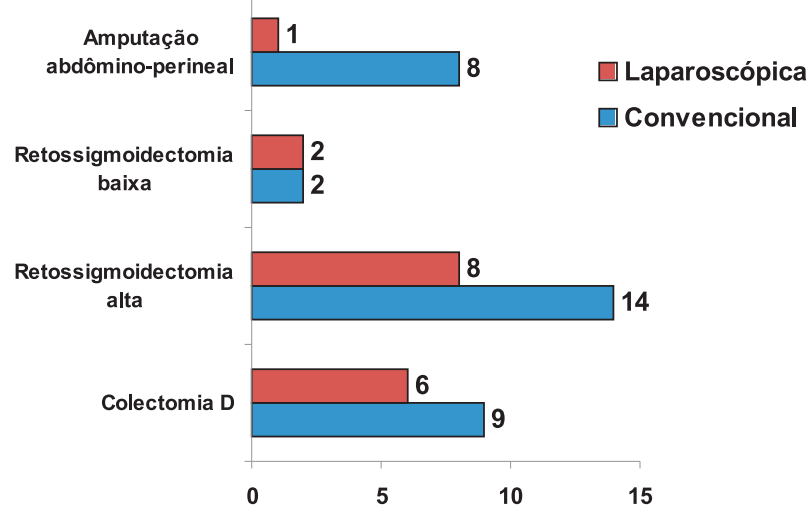

Figura 4 - Distribuição dos tipos de ressecção cirúrgica quanto à topografia dos tumores em relação às vias de acesso.

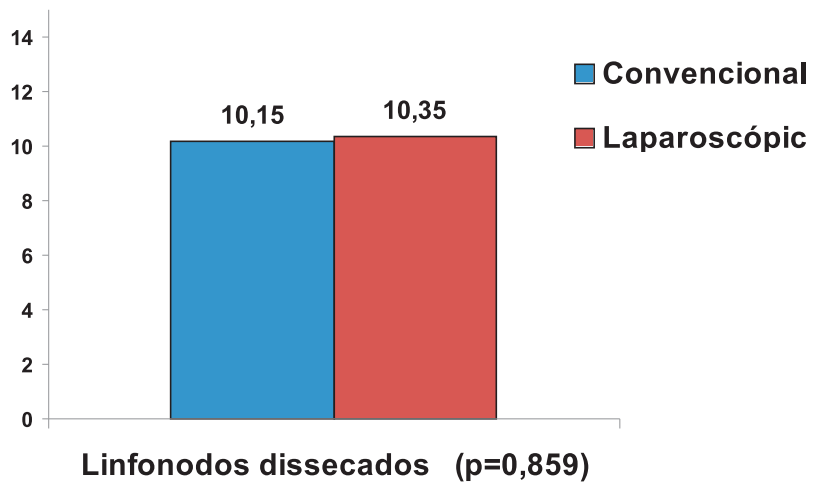

Figura 5 - Número médio de linfonodos dissecados entre as vias de acesso, análise estatística pelo método de Mann-Whitney.

rurgia convencional, além dos benefícios do pós-operatório a curto e longo prazo $^{10-13}$.

Os princípios oncológicos da ressecção do CCR foram descritos por muitos autores. A ligadura dos vasos mesocólicos nas suas raízes, correspondentes ao local do tumor, definindo-se uma linfadenectomia ampla em toda a topografia da lesão, é um princípio básico em cirurgia oncológica, e deve ser sempre seguido no tratamento do CCR, independentemente de sua via de acesso. Na curva inicial de aprendizado em ressecções colorretais por videolaparoscopia, alguns trabalhos demonstraram que o tratamento das neoplasias deveria ser precedido por uma experiência prévia no tratamento de doenças benignas, como diverticulite e adenomas colorretais, por exemplo.

Presume-se que uma ressecção por CCR por laparoscopia seja bem realizada, do ponto de vista oncológico, se a linfadenectomia for bem realizada, entre outros princípios técnicos. Portanto, este trabalho procurou analisar se a linfadenectomia realizada por laparoscopia foi equivalente à por via convencional, em uma curva inicial de aprendizado, num serviço universitário de ensino em coloproctologia.

Nesta casuística, poucas diferenças entre os grupos estudados (cirurgia convencional e videolaparoscópica) foram observadas. O número de pacientes submetidos a ressecções convencionais foi maior do que por videolaparoscopia (33 vs. 17 pacientes). Uma justificativa para este fato baseia-se na seleção de pacientes portadores de CCR para a técnica laparoscópica, uma vez que se tratava de um início de curva de aprendizado. Com isso, tumores maiores, pacientes obesos e portadores de qualquer fator que levasse à maior dificuldade técnica (comorbidades, por exemplo), eram direcionados para cirurgia convencional. No entanto, presume-se que no decorrer dos anos, esta relação será invertida, visto que depois de certa experiência adquirida, todos os pacientes com CCR serão abordados por videolaparoscopia, exceto tumores T4, pacientes com contra-indicações ao pneumoperitônio ou portadores de outras comorbidades que contraindiquem esta via de acesso.

A média de idade encontrada neste estudo foi maior no grupo da cirurgia convencional (62,84 anos) em comparação ao grupo da cirurgia laparoscópica (52,23 anos). Este achado é diferente do que o encontrado nas maiores séries da literatura, que mostram médias de idade semelhantes ${ }^{10-13}$. O número ainda limitado de pacientes desta casuística, associado ao fato de se tratar de um estudo retrospectivo em início da curva de aprendizado, são fatores que refletem a seleção criteriosa dos pacientes mais jovens para ressecção laparoscópica, visto que estes toleram maior tempo de pneumoperitônio sem efeitos hemodinâmicos adversos, além de apresentarem menores comorbidades clínicas.

$\mathrm{O}$ estadiamento analisado neste estudo foi o de Dukes, e não o TNM. A justificativa para este fato baseia-se na metodologia de análise do laudo da patologia dos espécimes de ressecção colorretal. Como não se analisou detalhadamente os prontuários completos dos pacientes (exames de tomografia, entre outros), a análise retrospectiva de metástases a distância poderia ser prejudicada no exame do laudo das peças isoladamente. Ao se comparar as duas técnicas, não se encontrou diferenças no número de pacientes com tumores Dukes A (4 pacientes para cada via de acesso). Este fato pode ser justificado pelo encorajamento de 
Análise do Número de Linfonodos em Espécimes de Ressecções Colorretais por Neoplasia entre a Cirurgia Aberta e Videolaparoscópica Paulo Gustavo Kotze e Cols. se operar tumores menores por videolaparoscopia. Já nos pacientes com tumores Dukes B e C, houve predomínio do número de casos na via convencional. Este predomínio foi mais intenso nos pacientes com tumores classificados como Dukes $\mathrm{C}$, (16 pacientes operados por acesso convencional contra 6 por acesso laparoscópico). Estes pacientes geralmente apresentam tumores maiores, com maior acometimento linfonodal, e foram previamente selecionados para cirurgia aberta, para que na curva de aprendizado, não houvesse prejuízo oncológico na videocirurgia.

Dentre os procedimentos realizados, houve predomínio da colectomia direita $(n=6)$ e da retossigmoidectomia com anastomose alta $(n=8)$ nas ressecções laparoscópicas. Uma justificativa evidente para estes números é baseada na maior facilidade técnica destas operações, se comparadas às intervenções sobre o reto previamente irradiado (retossigmoidectomias com anastomoses baixas e amputações do reto). Os trabalhos randomizados igualmente demonstram que as operações sobre o reto são mais difíceis de serem realizadas do ponto de vista técnico, e devem ser realizadas após uma determinada curva de aprendizado. Esta casuística, portanto, seguiu estes princípios da literatura ${ }^{5,6,21}$.

O principal objetivo deste estudo foi analisar a amostragem linfonodal encontrada nos espécimes cirúrgicos destes grupos. A média de linfonodos encontrada foi de 10,35 nos espécimes ressecados por via laparoscópica e 10,15 nos ressecados por via convencional, sem significância estatística $(p=0,859)$. Não houve, portanto, diferença entre as duas vias de acesso, o que comprova a qualidade oncológica da laparoscopia no início desta curva de aprendizado.

Entretanto, a média de linfonodos dissecados do presente estudo é inferior ao número mínimo de 12 linfonodos preconizado pelas principais diretrizes oncológicas $^{5,6,21}$. Uma provável razão para estes achados pode ser explicada pela inclusão nesta casuística de alguns casos de resseções de tumores do reto médio e distal, previamente irradiados. Alguns estudos evidenciam que a neoadjuvância realizada para estes tumores podem reduzir o número de linfonodos dissecados nas peças cirúrgicas. $\mathrm{O}$ tratamento pré-operatório pode ainda esterilizar linfonodos previamente acometidos $^{22}$. Esta foi uma das grandes limitações do presente estudo, além da metodologia retrospectiva e amostragem pequena de pacientes: a análise conjunta de tumores cólicos e de tumores do reto.
Há estudos que evidenciam que a remoção de mais de 18 linfonodos correlaciona-se com uma melhor sobrevida ${ }^{23,24}$. O assunto realmente é controverso, mas sabe-se que o real benefício da linfadenectomia, além do efeito terapêutico, é o estadiamento correto para que o paciente seja beneficiado pela quimioterapia adjuvante, o que apresenta verdadeiro impacto na sobrevida ${ }^{25}$. Jacob e Salky mostraram ainda que a média de 10 linfonodos não afetou a sobrevida dos pacientes em 5 anos de sobrevida, comparando-se os grupos submetidos a colectomias abertas e laparoscópicas ${ }^{26}$

É importante salientar que o número de linfonodos identificados pelos patologistas nem sempre corresponde ao número de linfonodos dissecados pelo cirurgião. $\mathrm{O}$ estudo randomizado COLOR, por exemplo, apresentou a média de 10 linfonodos dissecados em ambos os grupos ${ }^{14}$. O estudo de Barcelona, registrou uma média de 11,1 linfonodos nos 2 grupos $^{11}$. O estudo $\operatorname{COST}^{12}$ obteve média de 12 linfonodos nos 2 grupos e o estudo CLASSIC ${ }^{13}$ a média foi 13,5 para o grupo laparoscópico e 12 para o grupo convencional, mas sem significância estatística. Um comparativo entre os principais estudos que analisaram o número de linfonodos entre as duas vias de acesso com o presente estudo está ilustrado na tabela 1.

Vários fatores podem subestimar o estadiamento patológico das peças ressecadas. A minuciosidade e o método de detecção de linfonodos realizado pelo patologista são fatores cruciais. Na maioria dos trabalhos da literatura, e também no presente estudo, os patologistas não foram estimulados a fazer uma análise acurada das peças. Para se obter uma dissecção fidedigna, é necessário laboriosa tarefa de dissecção pelo método visual e tátil, inclusive dos pequenos linfonodos (menores que $5 \mathrm{~mm}$ de diâmetro), os quais podem ser frequentemente acometidos por metástases ${ }^{27}$.

Alguns métodos podem ser utilizados para se otimizar a pesquisa por linfonodos nos espécimes de resseções por CCR. Em nosso meio, o uso de solução reveladora de linfonodos no estadiamento do câncer colorretal foi descrito por Araújo et al. Estes autores, com o uso de solução reveladora no preparo das peças, conseguiram aumentar a média de linfonodos dissecados de 16,73 para 28,97 ( $p<0,001$ ), em comparação com as peças analisadas sem qualquer preparo, pelo mesmo grupo de patologistas ${ }^{28}$.

Kerwel et al. demonstraram importante benefício em uma mais acurada análise do número de 
Tabela 1 - Número de linfonodos dissecados nos principais estudos da literatura, em comparação com o presente trabalho.

\begin{tabular}{lcc}
\hline Autor (ano) & \multicolumn{2}{c}{ Número de linfonodos dissecados } \\
\cline { 2 - 3 } & Convencional & Laparoscópica \\
\hline Lacy et al. (Barcelona, 2002) $^{11}$ & 11,1 & 11,1 \\
Estudo COST, 2004 $^{12}$ & 12 & 12 \\
Veldkamp et al.(COLOR, 2005) $^{14}$ & 10 & 10 \\
Guillou et al. (CLASSIC, 2005) $^{13}$ & 13,5 & 12 \\
Kotze et al. (2010) - presente estudo $^{13}$ & 10,15 & 10,35 \\
\hline
\end{tabular}

linfonodos através da injeção de azul de metileno na raiz da artéria mesentérica inferior das peças cirúrgicas, após as ressecções, no câncer retal. Ao se comparar o grupo com corante injetado com o grupo sem corante, em um grupo total de 50 pacientes, o número de linfonodos no grupo corado foi de 30 +/13,5 contra $17+/-11$ no grupo sem a injeção do azul de metileno $(p<0,001)$. Os autores concluíram que a injeção do corante ajuda na melhor acurácia de análise do número de linfonodos, facilitando o trabalho dos patologistas na busca por nódulos para serem analisados ${ }^{29}$.

A ampla variação no número de linfonodos dissecados registrada na literatura, assim como a freqüente média inferior ao número mínimo exigido pelas diretrizes oncológicas, favorecem uma maximização de esforços tanto na técnica cirúrgica adotada pelo cirurgião, quanto na análise refinada do espécime pelo patologista. Esta correta preocupação tem o intuito de se evitar o subestadiamento patológi- co, com conseqüente melhora da sobrevida dos pacientes portadores de CCR.

\section{CONCLUSÕES}

Não houve diferença estatística entre o número de linfonodos analisados em espécimes de ressecções por câncer colorretal entre as vias convencional e laparoscópica. Estes achados permitem afirmar que na fase inicial da curva de aprendizado, houve equivalência oncológica entre os dois grupos.

O número médio de linfonodos nos dois grupos foi inferior ao preconizado pelas diretrizes, porém compatível com a maioria dos achados de séries de casos da literatura. Houve preponderância de colectomias direitas e retossigmoidectomias com anastomoses altas. Houve maior número de pacientes portadores de tumores avançados (Dukes B e C) no grupo de via de acesso convencional, na curva inicial de aprendizado.

\footnotetext{
ABSTRACT: Introduction: pathology staging with the analysis of the number of retrieved lymph nodes is an important factor in oncologic safety of colorectal cancer resections, in conventional and laparoscopic surgery. In the beginning of a learning curve in laparoscopy, equivalence between these two types of approaches can be compromised. The purpose of this study was to evaluate the number of retrieved lymph nodes in colorectal cancer resection specimens between conventional and laparoscopic surgery, and verify oncological equivalence between these techniques. Method: retrospective analysis of a case series of colorectal cancer specimens operated with conventional and laparoscopic surgery. Age, gender, type of operation, approach, staging and number of retrieved lymph nodes were analyzed. Statistical analysis with Mann-Whitney method was performed. Results: 50 patients were operated in the study period ( 33 with conventional and 17 with laparoscopic approach). Right hemicolectomy and high rectosigmoid resection were the most common procedures performed. The mean number of retrieved lymph nodes was 10,35 in the laparoscopy group and 10,15 in the conventional approach $(p=0,859)$. Conclusions: there was no statistical difference between the number of retrieved lymph nodes in colorectal cancer resection specimens between laparoscopic and conventional approach, in the beginning of a learning curve in laparoscopy.
}

Key words: Colorectal Neoplasia; Lymph Nodes; Colorectal Surgery, Laparoscopy. 
Análise do Número de Linfonodos em Espécimes de Ressecções Colorretais por Neoplasia entre a Cirurgia Aberta e Videolaparoscópica Paulo Gustavo Kotze e Cols.
Vol. 30

\section{REFERÊNCIAS}

1. Inca, Estimativa 2010: Incidência de Câncer no Brasil. Instituto Nacional do Câncer. [cited 2010 Jan 18]; Available from: http://www.inca.gov.br/estimativa/2010/index.asp? link $=$ conteudo_view.asp $\&$ ID $=5$.

2. Surveillance epidemiology and end results (SEER). National Cancer Intitute. 2010 [cited 2010 jan 19]; Available from: http:/ /www.seer.cancer.gov/statfacts/html/colorect.html.

3. Boland CR, Sinicrope FA, Brenner DE, Carethers JM. Colorectal cancer prevention and treatment. Gastroenterology. 2000 Feb;118(2 Suppl 1):S115-28.

4. Compton C, Fenoglio-Preiser CM, Pettigrew N, Fielding LP. American Joint Committee on Cancer Prognostic Factors Consensus Conference: Colorectal Working Group. Cancer. 2000 Apr 1;88(7):1739-57.

5. Engstrom PF, Arnoletti JP, Benson AB, Chen YJ, Choti MA, Cooper HS, et al. NCCN Clinical Practice Guidelines in Oncology: colon cancer. J Natl Compr Canc Netw. 2009 Sep;7(8):778-831.

6. Engstrom PF, Arnoletti JP, Benson AB, Chen YJ, Choti MA, Cooper HS, et al. NCCN Clinical Practice Guidelines in Oncology: rectal cancer. J Natl Compr Canc Netw. 2009 Sep;7(8):838-81.

7. Johnson A. Laparoscopic surgery. Lancet. 1997 Mar 1;349(9052):631-5.

8. Jacobs M, Verdeja JC, Goldstein HS. Minimally invasive colon resection (laparoscopic colectomy). Surg Laparosc Endosc. 1991 Sep;1(3):144-50.

9. Lacy AM, Garcia-Valdecasas JC, Delgado S, Grande L, Fuster J, Tabet J, et al. Postoperative complications of laparoscopicassisted colectomy. Surg Endosc. 1997 Feb;11(2):119-22.

10. Berends FJ, Kazemier G, Bonjer HJ, Lange JF. Subcutaneous metastases after laparoscopic colectomy. Lancet. $1994 \mathrm{Jul}$ 2;344(8914):58.

11. Lacy AM, Garcia-Valdecasas JC, Delgado S, Castells A, Taura P, Pique JM, et al. Laparoscopy-assisted colectomy versus open colectomy for treatment of non-metastatic colon cancer: a randomised trial. Lancet. 2002 Jun 29;359(9325):2224-9.

12. A comparison of laparoscopically assisted and open colectomy for colon cancer (COST Study). N Engl J Med. 2004 May 13;350(20):2050-9.

13. Guillou PJ, Quirke P, Thorpe H, Walker J, Jayne DG, Smith AM, et al. Short-term endpoints of conventional versus laparoscopic-assisted surgery in patients with colorectal cancer (MRC CLASICC trial): multicentre, randomised controlled trial. Lancet. 2005 May 14-20;365(9472):1718-26.

14. Veldkamp R, Kuhry E, Hop WC, Jeekel J, Kazemier G, Bonjer $\mathrm{HJ}$, et al. Laparoscopic surgery versus open surgery for colon cancer: short-term outcomes of a randomised trial. Lancet Oncol. 2005 Jul;6(7):477-84.

15. Aziz O, Constantinides V, Tekkis PP, Athanasiou T, Purkayastha S, Paraskeva P, et al. Laparoscopic versus open surgery for rectal cancer: a meta-analysis. Ann Surg Oncol. 2006 Mar;13(3):413-24.

16. Leroy J, Jamali F, Forbes L, Smith M, Rubino F, Mutter D, et al. Laparoscopic total mesorectal excision (TME) for rectal cancer surgery: long-term outcomes. Surg Endosc. 2004 Feb;18(2):281-9.

17. COLOR II: A randomized clinical trial comparing laparoscopic and open surgery for rectal cancer. Dan Med Bull. 2009;56(2):89-91.

18. Tekkis PP, Senagore AJ, Delaney CP, Fazio VW. Evaluation of the learning curve in laparoscopic colorectal surgery: comparison of right-sided and left-sided resections. Ann Surg. $2005 \mathrm{Jul} ; 242(1): 83-91$.

19. Habr-Gama A, Da Silva e Souza Jr A, Araujo S. Acesso vídeolaparoscópico no tratamento cirúrgico do câncer colorretal: análise crítica. Rev Ass Med Brasil. 1997;43(4):3526.

20. Pinho M, Ferreira L, Kleinubing Júnior H, et al. Câncer colorretal: análise do valor prognóstico do número total de linfonodos identificados na peça de ressecção. Rev Bras Coloproct. 2004;24(2):126-30.

21. Nelson H, Petrelli N, Carlin A, Couture J, Fleshman J, Guillem J, et al. Guidelines 2000 for colon and rectal cancer surgery. J Natl Cancer Inst. 2001 Apr 18;93(8):583-96.

22. Habr-Gama A, Perez RO, Proscurshim I, Rawet V, Pereira $\mathrm{DD}$, Sousa AH, et al. Absence of lymph nodes in the resected specimen after radical surgery for distal rectal cancer and neoadjuvant chemoradiation therapy: what does it mean? Dis Colon Rectum. 2008 Mar;51(3):277-83.

23. Prandi M, Lionetto R, Bini A, Francioni G, Accarpio G, Anfossi A, et al. Prognostic evaluation of stage B colon cancer patients is improved by an adequate lymphadenectomy: results of a secondary analysis of a large scale adjuvant trial. Ann Surg. 2002 Apr;235(4):458-63.

24. Le Voyer TE, Sigurdson ER, Hanlon AL, Mayer RJ, Macdonald JS, Catalano PJ, et al. Colon cancer survival is associated with increasing number of lymph nodes analyzed: a secondary survey of intergroup trial INT-0089. J Clin Oncol. 2003 Aug 1;21(15):2912-9.

25. Polignano F, Henderson N, Alishahi SM, Zito A. Laparoscopic colectomy for cancer and adequate lymphadenectomy: association between survival and number of lymph nodes. Surg Endosc. 2006 Jun;20(6):996-7.

26. Jacob BP, Salky B. Laparoscopic colectomy for colon adenocarcinoma: an 11-year retrospective review with 5-year survival rates. Surg Endosc. 2005 May;19(5):643-9.

27. Herrera-Ornelas L, Justiniano J, Castillo N, Petrelli NJ, Stulc JP, Mittelman A. Metastases in small lymph nodes from colon cancer. Arch Surg. 1987 Nov;122(11):1253-6.

28. Araujo S, Cabral M, Lacerda Filho A, Horta J, Luz M, Silva R. Impacto do Uso da Solução Reveladora de Linfonodos no Estadiamento do Câncer Colorretal. Rev Bras Coloproct. 2009;29(3):279-86. 
29. Kerwel TG, Spatz J, Anthuber M, Wunsch K, Arnholdt H, Markl B. Injecting methylene blue into the inferior mesenteric artery assures an adequate lymph node harvest and eliminates pathologist variability in nodal staging for rectal cancer. Dis Colon Rectum. 2009 May;52(5):935-41.
Endereço para correspondência:

PAULO GUSTAVO KOTZE

Rua Jaime Balão, 975 - ap. 106 - Hugo Lange

Curitiba - PR

CEP: 80040-340

Fone/FAX: (41) 3022-5500

E-mail:pgkotze@hotmail.com 